\title{
Resonant excitation of coastal Kelvin waves in the two-layer rotating shallow water model
}

\author{
V. Zeitlin \\ Institut Universitaire de France, Laboratoire de Météorologie Dynamique, UMR8539, CNRS - University P. and M. Curie, \\ Paris, France
}

Correspondence to: V. Zeitlin (zeitlin@1md.ens.fr)

Received: 23 August 2013 - Revised: 14 October 2013 - Accepted: 16 October 2013 - Published: 19 November 2013

\begin{abstract}
Resonant excitation of coastal Kelvin waves by free inertia-gravity waves impinging on the coast is studied in the framework of the simplest baroclinic model: two-layer rotating shallow water with an idealized straight coast. It is shown that, with respect to the previous results obtained with the one-layer model, new resonances leading to a possible excitation of Kelvin waves appear. The most interesting ones, described in the paper, are resonances of a baroclinic inertiagravity wave with either another wave of this kind, or with a coastal current, leading to generation of a barotropic Kelvin wave. A forced Hopf equation results in any case for the evolution of the Kelvin wave amplitude.
\end{abstract}

\section{Introduction}

The present work is a development of the results of the paper by Reznik and Zeitlin (2009) where a mechanism of resonant excitation of the coastal Kelvin waves by inertia-gravity waves was shown to exist in the simplest rotating shallow water model with an idealized straight coast. The main observation below is that including baroclinic effects again in the simplest two-layer rotating shallow water model allows for new resonances leading to the excitation of Kelvin waves by inertia-gravity waves impinging on the coast. Probably the most interesting of these new resonances is the one including an inertia-gravity wave, a Kelvin wave and a coastal current. Coastal currents being ubiquitous in the ocean, such a resonance provides a mechanism of universal nature for Kelvin wave excitation, which to our knowledge was previously unnoticed in the literature. The process of excitation of Kelvin waves which are dispersionless and thus prone to breaking, with formation of the zones of enhanced mixing and dissipation in the coastal zones, is of potential importance for understanding the routes to dissipation in the ocean.

The paper is organized as follows. In Sect. 2 we present the model and deduce its wave spectrum. In Sect. 3 we sketch the resonances leading to the excitation of Kelvin waves and develop in some detail the cases of the barotropic Kelvin wave excitation by a baroclinic inertia-gravity wave interacting with another one, or with a coastal current. Section 4 contains the conclusions and discussion.

\section{The model and its wave spectrum}

The equations of the two-layer rotating shallow water model, which is schematically represented in Fig. 1, are:

$$
\begin{aligned}
\frac{D_{1}}{D t} \boldsymbol{v}_{1}+f \hat{z} \wedge \boldsymbol{v}_{1}+g \nabla\left(h_{1}+h_{2}\right) & =0, \\
\partial_{t} h_{1}+\nabla \cdot\left(h_{1} \boldsymbol{v}_{1}\right) & =0, \\
\frac{D_{2}}{D t} \boldsymbol{v}_{2}+f \hat{z} \wedge \boldsymbol{v}_{2}+g \nabla\left(r h_{1}+h_{2}\right) & =0, \\
\partial_{t} h_{2}+\nabla \cdot\left(h_{2} \boldsymbol{v}_{2}\right) & =0 .
\end{aligned}
$$

Here $\boldsymbol{v}_{i}=\left(u_{i}, v_{i}\right), i=1,2$ are the velocities in the respective layers (layer 1 on top of layer 2), $h_{i}$ are the thicknesses of the layers with unperturbed values $H_{i}, r=\frac{\rho_{1}}{\rho_{2}} \leq 1$ is the density ratio of the layers characterizing stratification, $f=$ const $>0$ (Northern Hemisphere) is the Coriolis parameter, and $g$ is the gravity acceleration. $\frac{D_{i}}{D t}$ denote the advective derivatives with corresponding velocities: $\frac{D_{i}}{D t}=\partial_{t}+\boldsymbol{v} \cdot \nabla$.

By introducing the time scale $f^{-1}$, the velocity scale $U$, the vertical scale $H=H_{1}+H_{2}$ and corresponding dimensionless unperturbed thicknesses $\frac{H_{1}}{H}=d, \frac{H_{2}}{H}=1-d, d<1$, the horizontal scale $L \sim \frac{g H}{f^{2}}$, the Rossby number $R o=\epsilon=$ $\frac{U}{f L}$, which will be supposed to be small in what follows, and assuming that typical perturbations $\eta_{1}, \eta_{2}$ of the thicknesses 


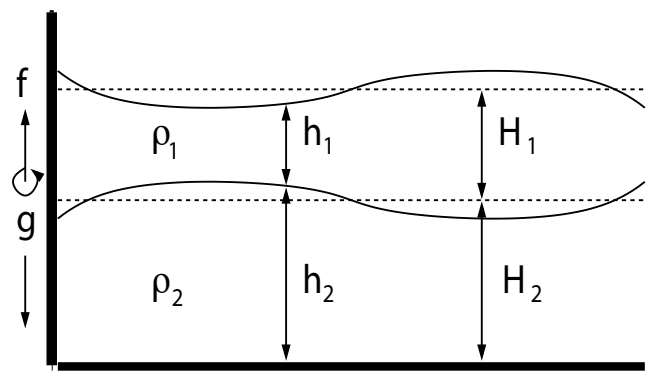

Fig. 1. A side view of the two-layer rotating shallow water model with a straight coast on the $f$ plane.

of the layers are $\mathcal{O}(\epsilon)$, we obtain from Eq. (1) the following non-dimensional equations:

$$
\begin{aligned}
\left(\partial_{t}+\epsilon \boldsymbol{v}_{1} \cdot \nabla\right) \boldsymbol{v}_{1}+f \hat{z} \wedge \boldsymbol{v}_{1}+\nabla\left(\eta_{1}+\eta_{2}\right) & =0, \\
\partial_{t} \eta_{1}+\nabla \cdot\left(\left(d+\epsilon \eta_{1}\right) \boldsymbol{v}_{1}\right) & =0, \\
\left(\partial_{t}+\epsilon \boldsymbol{v}_{2} \cdot \nabla\right) \boldsymbol{v}_{2}+f \hat{z} \wedge \boldsymbol{v}_{2}+\nabla\left(r \eta_{1}+\eta_{2}\right) & =0, \\
\partial_{t} \eta_{2}+\nabla \cdot\left(\left(1-d+\epsilon \eta_{2}\right) \boldsymbol{v}_{2}\right) & =0 .
\end{aligned}
$$

For illustrative purposes, in what follows we will choose the most simple case, $d=\frac{1}{2}$, although calculations may be carried out, with some more effort, for an arbitrary $d$.

The linear part of the equations (2), remaining after taking the limit $\epsilon \rightarrow 0$, may be factorized by means of decomposition into barotropic (+) and baroclinic (-) components, which reads in the case $d=\frac{1}{2}$ :

$\boldsymbol{v}^{ \pm}=\sqrt{r} \boldsymbol{v}_{1} \pm \boldsymbol{v}_{2}, \quad \eta^{ \pm}=2\left(\sqrt{r} \eta_{1} \pm \eta_{2}\right)$,

where we introduced an additional factor 2 in the second equation for technical convenience.

In terms of the baroclinic and the barotropic components (3), Eq. (2) becomes

$$
\begin{aligned}
\partial_{t} \boldsymbol{v}^{+} & +\hat{z} \wedge \boldsymbol{v}^{+}+\frac{1+\sqrt{r}}{2} \nabla \eta^{+}=-\frac{\epsilon}{4}\left[\left(\frac{1}{\sqrt{r}}+1\right)\left(\boldsymbol{v}^{+} \cdot \nabla \boldsymbol{v}^{+}+\boldsymbol{v}^{-} \cdot \nabla \boldsymbol{v}^{-}\right)\right. \\
& \left.+\left(\frac{1}{\sqrt{r}}-1\right)\left(\boldsymbol{v}^{+} \cdot \nabla \boldsymbol{v}^{-}+\boldsymbol{v}^{-} \cdot \nabla \boldsymbol{v}^{+}\right)\right] \\
\partial_{t} \eta^{+} & +\nabla \cdot \boldsymbol{v}^{+}=-\frac{\epsilon}{4}\left[\left(\frac{1}{\sqrt{r}}+1\right) \boldsymbol{\nabla} \cdot\left(\eta^{+} \boldsymbol{v}^{+}+\eta^{-} \boldsymbol{v}^{-}\right)\right. \\
& \left.+\left(\frac{1}{\sqrt{r}}-1\right) \nabla \cdot\left(\eta^{+} \boldsymbol{v}^{-}+\eta^{-} \boldsymbol{v}^{+}\right)\right] \\
\partial_{t} \boldsymbol{v}^{-} & +\hat{z} \wedge \boldsymbol{v}^{-}+\frac{1-\sqrt{r}}{2} \nabla \eta^{-}=-\frac{\epsilon}{4}\left[\left(\frac{1}{\sqrt{r}}-1\right)\left(\boldsymbol{v}^{+} \cdot \nabla \boldsymbol{v}^{+}+\boldsymbol{v}^{-} \cdot \nabla \boldsymbol{v}^{-}\right)\right. \\
& \left.+\left(\frac{1}{\sqrt{r}}+1\right)\left(\boldsymbol{v}^{+} \cdot \nabla \boldsymbol{v}^{-}+\boldsymbol{v}^{-} \cdot \nabla \boldsymbol{v}^{+}\right)\right] \\
\partial_{t} \eta^{-} & +\nabla \cdot \boldsymbol{v}^{-}=-\frac{\epsilon}{4}\left[\left(\frac{1}{\sqrt{r}}-1\right) \boldsymbol{\nabla} \cdot\left(\eta^{+} \boldsymbol{v}^{+}+\eta^{-} \boldsymbol{v}^{-}\right)\right. \\
& \left.+\left(\frac{1}{\sqrt{r}}+1\right) \boldsymbol{\nabla} \cdot\left(\eta^{+} \boldsymbol{v}^{-}+\eta^{-} \boldsymbol{v}^{+}\right)\right]
\end{aligned}
$$

We will consider the system (1) in the semi-infinite plane with an idealized straight coast. The $y$ axis of the coordinate system will be directed along the coast, and the $x$ axis

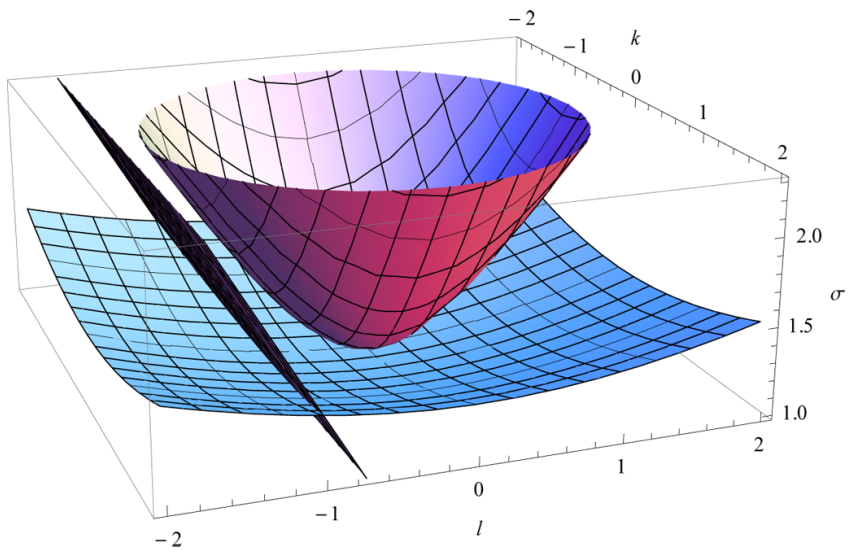

Fig. 2. Dispersion relation $\sigma(k, l)$ for the waves in the 2-layer system at $r=0.5$. Upper surface: barotropic IG waves, lower surface: baroclinic IG waves. Inclined plane: barotropic Kelvin waves. An inclined plane with lesser slope, corresponding to the baroclinic Kelvin waves, is not displayed beneath the dispersion surface for baroclinic IG, to avoid overcharging the figure.

out from the coast. As follows from Eqs. (4)-(7), in the linear limit $\epsilon \rightarrow 0$ the system decouples into two rotating shallow water subsystems (the barotropic and the baroclinic ones) with different dimensionless effective gravities $\frac{1 \pm \sqrt{r}}{2}$, respectively. The wave spectrum of rotating shallow water in the semi-infinite domain is well known, cf. e.g. Gill (1982). It consists of incident and is reflected by the coast barotropic (upper sign) or baroclinic (lower sign) inertia-gravity (IG) waves with the dispersion relation:

$\sigma_{\mathrm{IG}^{ \pm}}^{2}=1+c_{ \pm}^{2} \boldsymbol{k}^{2}$

where $\sigma$ is the wave frequency and $\boldsymbol{k}=(k, l)$ is the wavevector, and dispersionless Kelvin $(\mathrm{K})$ waves propagating along the $y$ axis towards lesser $y$ with the phase velocity $c_{ \pm}$:

$\sigma_{\mathrm{K}^{ \pm}}^{2}=c_{ \pm}^{2} l^{2}$,

and decaying with $x$ as $e^{-x / c_{ \pm}}$, respectively. We introduced here the dimensionless phase velocities of the baroclinic and barotropic gravity waves

$c_{ \pm}=\sqrt{\frac{1 \pm \sqrt{r}}{2}}$.

Graphical representation of dispersion relations of the waves in the system is given in Fig. 2.

The velocity and thickness fields for IG and $\mathrm{K}$ waves are given by:

$\left(u_{\mathrm{IG}}^{ \pm}, v_{\mathrm{IG}}^{ \pm}, \eta_{\mathrm{IG}}^{ \pm}\right)=\left(U^{ \pm}(x), V^{ \pm}(x), N^{ \pm}(x)\right) e^{i(l y-\sigma t)}+c . c .$,

and

$\left(u_{\mathrm{K}}^{ \pm}, v_{\mathrm{K}}^{ \pm}, \eta_{\mathrm{K}}^{ \pm}\right)=\left(0, c_{ \pm} \mathrm{K}^{ \pm}\left(y+c_{ \pm} t\right),-\mathrm{K}^{ \pm}\left(y+c_{ \pm} t\right)\right) e^{-x / c_{ \pm}}$, 
where $\mathrm{K}^{ \pm}$are arbitrary functions of their arguments $\xi^{ \pm}$defined as

$\xi^{ \pm}=y+c_{ \pm} t$

The boundary condition of vanishing normal velocity at the coast imposes

$U^{ \pm}=A\left(e^{i k x}-e^{-i k x}\right)$,

where $A$ is the (complex) wave amplitude, and the expressions for $V$ and $N$ (polarisation relations) follow from the linearized Eqs. (4)-(7):

$V^{ \pm}=A\left(1+c_{ \pm}^{2} l^{2}\right)\left(\frac{e^{i k x}}{i \sigma^{ \pm}+l k c_{ \pm}^{2}}+\frac{e^{-i k x}}{-i \sigma^{ \pm}+l k c_{ \pm}^{2}}\right)$,

$N^{ \pm}=A\left(\frac{i k+\sigma_{ \pm} l}{c_{ \pm}^{2} l k+i \sigma_{ \pm}} e^{i k x}-\frac{i k-\sigma_{ \pm} l}{c_{ \pm}^{2} l k-i \sigma_{ \pm}} e^{-i k x}\right)$,

where we assume that $k<0$.

\section{Resonant excitation of Kelvin waves in the two-layer system}

\subsection{Wave-wave resonances}

Let us recall of the principle of resonant excitation of waves in the present context. In general, if we consider the system (4)-(7) in the linear approximation, take a solution which consists of a superposition of waves, and try to calculate the nonlinear corrections, we generally encounter a problem of resonances. The nonlinear terms in the Eqs. (4)-(7) are resonant if the frequencies and wavevectors of any pair of initial waves obey the relations:

$\boldsymbol{k}_{1} \pm \boldsymbol{k}_{2}=\boldsymbol{k}, \quad \sigma\left(\boldsymbol{k}_{1}\right) \pm \sigma\left(\boldsymbol{k}_{2}\right)=\sigma(\boldsymbol{k})$

where $\sigma(\boldsymbol{k})$ follows the dispersion relation for linear waves in the system and the indices 1,2 (not to be confused with layer numbers) enumerate the resonating waves, whatever their nature (baroclinic or barotropic).

In the case of resonance, the forcing due to the zero-order fields in the equations for the first-order corrections projects onto the eigensolutions of the linear system and leads to the resonant growth of the corrections. As is well-known, this situation is treated by introducing slow modulations of the initial wave amplitudes. In the case of resonance, the amplitude of the wave with the wavevector $\boldsymbol{k}$ grows due to the resonance of the waves with wavenumbers $\boldsymbol{k}_{1,2}$. In what follows, we will be interested in resonant excitation of Kelvin waves, which are trapped near the coast by free inertiagravity waves which come from infinity and are reflected by the coast. Kelvin waves are non-propagative in the $x$ direction perpendicular to the coast, hence the resonance conditions include only the $y$ component of the wavevector in this case, cf. Reznik and Zeitlin (2009):

$l_{\mathrm{IG}_{1}} \pm l_{\mathrm{IG}_{2}}=l_{\mathrm{K}}, \quad \sigma_{\mathrm{IG}}\left(\boldsymbol{k}_{1}\right) \pm \sigma_{\mathrm{IG}}\left(\boldsymbol{k}_{2}\right)=\sigma_{\mathrm{K}}(l)$,

where, again, each wave may be either barotropic or baroclinic. We will refer below to the upper- and lower-sign cases in Eq. (18) as the "sum" and the "difference" resonances, respectively. The incident and reflected IG waves in this context may be thought of as a mode propagating in the $y$ direction.

Suppose that a pair of IG waves, whatever their character, is in resonance (18) with either a barotropic or a baroclinic $\mathrm{K}$ wave. These three waves constitute a zero-order solution which will be considered to be slow time-dependent, with the slow time $T=\epsilon t$ :

$$
\begin{aligned}
\left(u^{(0)}, v^{(0)}, \eta^{(0)}\right) & =\left(u_{\mathrm{K}}^{(0)}, v_{\mathrm{K}}^{(0)}, \eta_{\mathrm{K}}^{(0)}\right)(x, y, t, T) \\
& +\sum_{i=1,2}\left(u_{i_{\mathrm{IG}}}^{(0)}, v_{i_{\mathrm{IG}}}^{(0)}, \eta_{i_{\mathrm{IG}}}^{(0)}\right)(x, y, t, T),
\end{aligned}
$$

where the appropriate components, barotropic or baroclinic, are understood for each wave, depending on the precise nature of the resonance. For the first-order correction $\left(u^{(1) \pm}, v^{(1) \pm}, \eta^{(1)^{ \pm}}\right)(x, y, t, T)$ we get

$$
\begin{aligned}
u_{t}^{(1) \pm}-v^{(1) \pm}+c_{ \pm}^{2} \eta_{x}^{(1) \pm} & =-u_{T}^{(0) \pm}+\mathrm{NL}_{u}^{ \pm} \equiv R_{u}^{ \pm}, \\
v_{t}^{(1) \pm}+u^{(1) \pm}+c_{ \pm}^{2} \eta_{y}^{(1) \pm} & =-v_{T}^{(0) \pm}+\mathrm{NL}_{v}^{ \pm} \equiv R_{v}^{ \pm}, \\
\eta_{t}^{(1) \pm}+u_{x}^{(1) \pm}+v_{y}^{(1) \pm} & =-\eta_{T}^{(0) \pm}+\mathrm{NL}_{\eta}^{ \pm} \equiv R_{\eta}^{ \pm},
\end{aligned}
$$

where $\mathrm{NL}_{u, v, \eta}^{ \pm}$stand for corresponding nonlinear terms in Eqs. (4)-(7) built from the zero-order fields (19). As shown in Reznik and Zeitlin (2009), all essential information for Kelvin wave excitation is contained in the expressions $R_{v}^{ \pm}$ and $R_{\eta}^{ \pm}$. Up to non-resonant terms they have the form:

$$
\begin{aligned}
R_{v}^{ \pm} & =-c_{ \pm} \mathrm{K}_{T}^{ \pm} e^{-x / c_{ \pm}}-a_{ \pm} c_{ \pm}^{2} \mathrm{~K}^{ \pm} \mathrm{K}_{\xi^{ \pm}}^{ \pm} e^{-2 x / c_{ \pm}} \\
& -\left[\mathrm{NL}_{v}^{{ }^{\mathrm{IG}}} e^{i l \xi^{ \pm}}+\text {c.c. }\right], \\
R_{\eta}^{ \pm} & =\mathrm{K}_{T}^{ \pm} e^{-x / c_{ \pm}}+2 a_{ \pm} c_{ \pm} \mathrm{K}^{ \pm} \mathrm{K}_{\xi^{ \pm}}^{ \pm} e^{-2 x / c_{ \pm}} \\
& -\left[\mathrm{NL}_{\eta}^{{ }^{\mathrm{IG}}} e^{i l \xi^{ \pm}}+\text {c.c. }\right],
\end{aligned}
$$

where we introduced a useful notation for what follows:

$a_{ \pm}=\frac{1}{4}\left(\frac{1}{\sqrt{r}} \pm 1\right)$

and $\mathrm{NL}_{v, \eta}^{ \pm \mathrm{IG}}$ denote bilinear combinations proportional to $|A|^{2}$ and resulting from inserting into the nonlinear terms the zeroorder IG fields with polarization relations (14)-(16). To obtain the evolution equation for the Kelvin wave $\mathrm{K}^{ \pm}\left(\xi^{ \pm}, T\right)$, we follow Reznik and Zeitlin (2009) and subtract the third 
equation from the second one, multiplied by $c_{ \pm}^{-1}$ in Eq. (20). After multiplying by $e^{-x / c_{ \pm}}$and integrating in $x$, we get:

$$
\begin{gathered}
B_{t}^{ \pm}-c_{ \pm} B_{y}^{ \pm}=-\int_{0}^{\infty} \mathrm{d} x e^{-x / c_{ \pm}}\left(R_{\eta}^{ \pm}-c_{ \pm}^{-1} R_{v}^{ \pm}\right), \\
B^{ \pm}=\int_{0}^{\infty} \mathrm{d} x e^{-x / c_{ \pm}}\left(c_{ \pm}^{-1} v^{(1) \pm}-\eta^{(1) \pm}\right) .
\end{gathered}
$$

As follows from Eqs. (21) and (22), the r.h.s. of Eq. (24) is a function of $\xi^{ \pm}$only, and to guarantee the boundedness of $B^{ \pm}$it should be equal to zero. This is because the 1.h.s. contains the derivative of the function $B^{+}\left(B^{-}\right)$with respect to $t$ uniquely at fixed $\xi^{+}\left(\xi^{-}\right)$, and with the r.h.s. independent of $\xi^{-}\left(\xi^{+}\right)$, a linear growth of $B^{+}\left(B^{-}\right)$in $t$ results. Thus,

$\int_{0}^{\infty} \mathrm{d} x e^{-x / c_{ \pm}}\left(R_{\eta}^{ \pm}-c_{ \pm}^{-1} R_{v}^{ \pm}\right)=0$,

which leads to the evolution equation of the form:

$\mathrm{K}_{T}^{ \pm}+a_{ \pm} c_{ \pm} \mathrm{K}^{ \pm} \mathrm{K}_{\xi^{ \pm}}^{ \pm}=\mathcal{S}$,

where $\mathcal{S}$ is a forcing term produced by nonlinear interaction of IG waves, which is nonzero in general. As in Reznik and Zeitlin (2009), it is proportional to the product of amplitudes of the two IG waves, and is harmonic in $\xi^{ \pm}$. The detailed expression for $\mathcal{S}$ in terms of the wavenumbers of the resonating IG waves depends on the precise nature of the resonance and may be established, like in Reznik and Zeitlin (2009), after some algebra. The properties of the harmonically forced Hopf equation (26) may be found in the literature (cf. Reznik and Zeitlin, 2009 and references therein), the most important being formation of the enhanced mixing zones - Kelvin fronts Fedorov and Melville (1995) ${ }^{1}$. We should emphasize that even if the amplitude (envelope) of the Kelvin wave $\mathrm{K}^{ \pm}$ is initially zero, it will grow according to Eq. (26) and eventually break.

With respect to the one-layer case of Reznik and Zeitlin (2009), where only the "difference" resonances were operational, the "sum" resonances between the waves of the same and different kinds (barotropic and baroclinic) can lead to the resonance excitation of the Kelvin waves in the two-layer model. Thus, barotropic Kelvin waves can be excited by the "difference" resonances of two barotropic IG waves, as in the one-layer case, and by the "sum" resonances including either a baroclinic and a barotropic IG wave, or a pair of baroclinic IG waves. The baroclinic Kelvin waves may only be excited by "difference" resonances of all kinds (baroclinicbaroclinic, barotropic-baroclinic and barotropic-barotropic,

${ }^{1}$ Although we do not introduce explicit dissipation in our analysis, it becomes necessary for properly resolving the Kelvin-wave breaking events - a numerical dissipation is, normally, sufficient in numerical simulations.

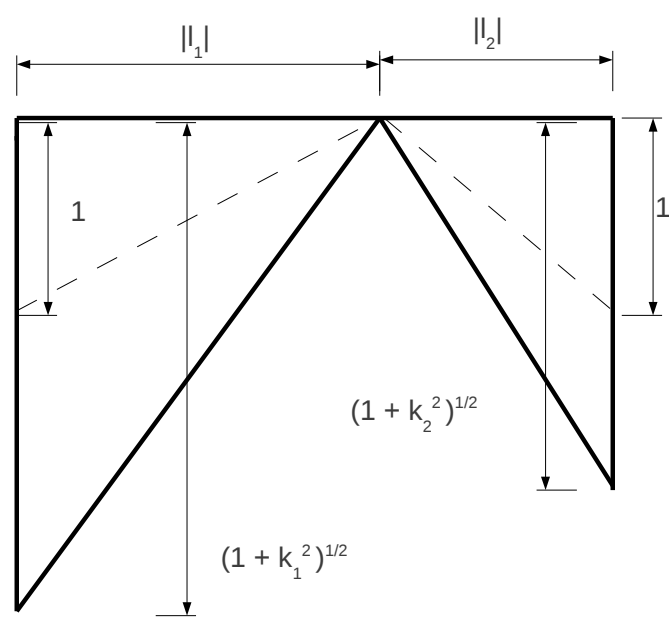

Fig. 3. Geometric representation of the resonance condition (31). Hypotenuses of the right-angle triangles (solid) represent the two entries in the 1.h.s., with their minimal values also shown (dashed).

LeCunuder, 2013). The "difference" resonances may be analysed along the lines of Reznik and Zeitlin (2009), so we sketch below only the mechanism of the sum resonance of two baroclinic IG waves generating a barotropic Kelvin wave.

The resonance conditions in question are, cf. Eq. (18):

$l_{\mathrm{IG}_{1}}+l_{\mathrm{IG}_{2}}=l_{\mathrm{K}}, \quad \sigma_{\mathrm{IG}}\left(\boldsymbol{k}_{1}\right)+\sigma_{\mathrm{IG}}\left(\boldsymbol{k}_{2}\right)=\sigma_{\mathrm{K}}(l)$.

We will omit the subscripts IG and $\mathrm{K}$ from now on. Equations (27), (8) and (9) give:

$l_{1}+l_{2}=l=-|l|$,

$$
\sqrt{1+c_{-}^{2}\left(k_{1}^{2}+l_{1}^{2}\right)}+\sqrt{1+c_{-}^{2}\left(k_{2}^{2}+l_{2}^{2}\right)}=c_{+}|l|,
$$

where, as usual, we adopt a convention of positive wave frequencies, which means that the wavenumber $l$ of the Kelvin wave is negative. The resonance condition on frequencies, after renormalizing the wavevectors, is as follows:

$\left(k_{i}, l_{i}\right)=c_{-}^{-1}\left(\tilde{k}_{i}, \tilde{l}_{i}\right), i=1,2$,

and, omitting the tildes, is:

$\sqrt{1+k_{1}^{2}+l_{1}^{2}}+\sqrt{1+k_{2}^{2}+l_{2}^{2}}=-\frac{c_{+}}{c_{-}}\left(l_{1}+l_{2}\right)$.

Suppose that $l_{1,2}$ are both negative: $l_{1,2}=-\left|l_{1,2}\right|$. Equation (30) then takes the form:

$\sqrt{1+k_{1}^{2}+\left|l_{1}\right|^{2}}+\sqrt{1+k_{2}^{2}+\left|l_{2}\right|^{2}}=+\frac{c_{+}}{c_{-}}\left(\left|l_{1}\right|+\left|l_{2}\right|\right)$,

and admits a simple geometric interpretation presented in Fig. 3.

At fixed $l_{1,2}$ the minimum value of the 1.h.s. of Eq. (31) is equal to $\sqrt{1+|l|_{1}^{2}}+\sqrt{1+|l|_{2}^{2}}>|l|_{1}+|l|_{2}$ (sum of the lengths 

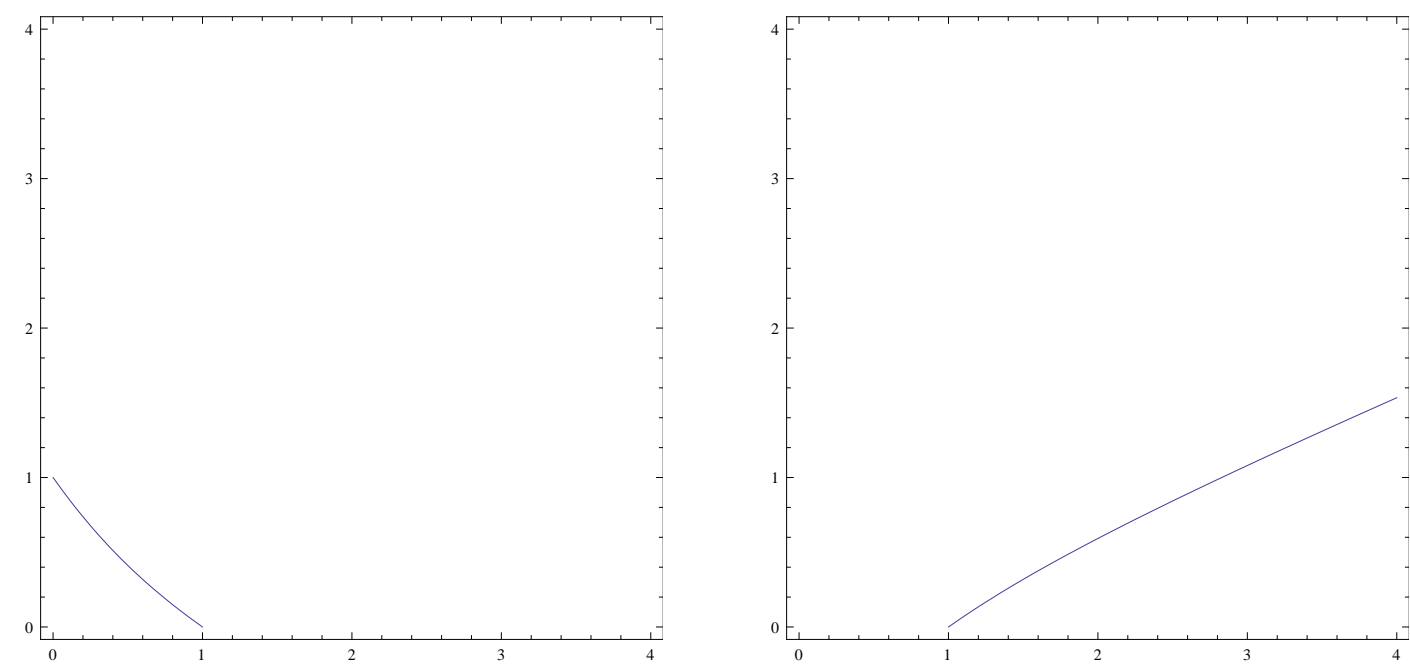

Fig. 4. Resonant region in the $|l|_{1},|l|_{2}$ plane for $r=0.5$. Left: both $l_{1,2}$ negative. Right: negative $l_{1}$ and positive $l_{2}$. Resonant wavenumbers are situated above the displayed limiting curve on the left panel, and beneath the limiting curve on the right panel. Horizontal (vertical) axis: $\left|l_{1}\right|\left(\left|l_{2}\right|\right)$. With growing $\frac{c_{+}}{c_{-}}$(growing $r$ ) the non-resonant region on the left panel shrinks, keeping the same form, while on the right panel it is the resonant region which is getting more and more narrow.

of the dashed intervals in Fig. 3 vs. the length of the horizontal interval). Thus if the ratio $\frac{c_{+}}{c_{-}}$is insufficiently large, $\frac{c_{+}}{c_{-}}<\frac{\sqrt{1+|l|_{1}^{2}}+\sqrt{1+|l|_{2}^{2}}}{|l|_{1}+|l|_{2}}$, i.e. the stratification is too strong, cf. Eq. (10), the resonance is not possible. Otherwise, as is easy to see from Fig. 3, one can always find $k_{1,2}$ to satisfy the resonance condition. Inversely, at fixed $\frac{c_{+}}{c_{-}}$, i.e. at a given stratification, the condition on $l_{1,2}$ for the existence of resonance is

$$
\frac{c_{+}}{c_{-}}\left(|l|_{1}+|l|_{2}\right)>\sqrt{1+|l|_{1}^{2}}+\sqrt{1+|l|_{2}^{2}} .
$$

Due to the $\sqrt{r}$ dependence, the resonance does not exist only for unrealistically small values of $r$. Analysis of Eq. (32) shows that, except for the values of $\frac{c_{+}}{c_{-}}$very close to 1 (very strong stratification), the resonance is always possible for high enough $|l|_{1,2}$. Similar analysis may be carried out in the case where one of the wavenumbers, say $l_{2}$, is positive. Typical regions of resonant $l_{1,2}$ are presented in Fig. 4 in both cases.

\subsection{Wave-meanflow resonance}

As mentioned in the Introduction, the two-layer system admits a new type of resonance leading to the excitation of Kelvin waves: IG wave-meanflow resonance. Indeed, a meanflow solution, which is, in fact, a solution of not only the linearized, but also of the full system, should be added to the solutions of the linear system described in Sect. 2. In non-dimensional terms it is given by

$\left\{\begin{aligned} u_{1_{\mathrm{M}}}=u_{2_{\mathrm{M}}} & =0, \\ -v_{1_{\mathrm{M}}}+\partial_{x}\left(\eta_{1_{\mathrm{M}}}+\eta_{2_{\mathrm{M}}}\right) & =0, \\ -v_{2_{\mathrm{M}}}+\partial\left(r \eta_{1_{\mathrm{M}}}+\eta_{2_{\mathrm{M}}}\right) & =0,\end{aligned}\right.$ and represents an along-coast current in geostrophic equilibrium. In terms of baroclinic and barotropic components we have for the mean current:

$u_{\mathrm{M}}^{ \pm}=0, \quad v_{\mathrm{M}}^{ \pm}=\frac{1 \pm \sqrt{r}}{2} \partial_{x} \eta_{\mathrm{M}}^{ \pm}$.

We will consider in what follows the functions $v_{1,2}$ rapidly decaying out of the coast (at $x=0$ ), and thus representing coastal currents, which are ubiquitous in the ocean. In the case of surface currents, probably the most relevant configuration, the lower layer is motionless:

$v_{2_{\mathrm{M}}}=0, \Rightarrow r \eta_{1_{\mathrm{M}}}+\eta_{2_{\mathrm{M}}}=0$.

As is known in another context (Reznik and Zeitlin, 2007), free waves can resonate with mean currents and thus excite trapped waveguide modes. A weak mean current along the waveguide (the coast in the present context) in such situation may be considered as a wave with zero frequency and zero wavenumber in the waveguide direction. For such a resonance to exist, the wave frequency and the wavenumber in the waveguide direction of the incoming free wave should coincide with those of the waveguide mode. From the intersection of the dispersion surfaces of the baroclinic IG waves and the barotropic Kelvin waves in Fig. 1, it is obvious that the resonance of a baroclinic IG wave, a mean current, and a barotropic Kelvin wave and, hence, resonant excitation of barotropic Kelvin waves via this resonance, is possible. We thus should have:

$\sigma_{\mathrm{IG}^{-}}=\sigma_{\mathrm{K}^{+}}, \quad l_{\mathrm{IG}^{-}}=l_{\mathrm{K}^{+}} \equiv l$,

i.e.

$c_{+}^{2} l^{2}=1+c_{-}^{2}\left(k^{2}+l^{2}\right)$, 
which leads to the following expression for the $x$-component $k$ of the wavenumber of the incoming IG wave:

$k^{2}=\left(\frac{c_{+}^{2}}{c_{-}^{2}}-1\right) l^{2}-\frac{1}{c_{-}^{2}}$.

Therefore, for a given stratification, i.e. a given ratio $\frac{c_{+}^{2}}{c^{2}}>$ 1 , the resonance is always possible for a sufficiently large negative $l$. With respect to the wave-wave resonance, the conditions of the present one are less restrictive, as it requires a single wave impinging on the coastal current, a ubiquitous situation in the ocean, and thus provides a universal mechanism for Kelvin wave excitation. We should stress that such a mechanism is impossible in the barotropic model, because the dispersion surface of the Kelvin waves always lies below the dispersion surface of the IG waves of the same type.

Let us see the details of this mechanism. We represent the zero-order flow in the form:

$$
\begin{aligned}
\left(u^{(0)}, v^{(0)}, \eta^{(0)}\right) & =\left(u_{\mathrm{K}}^{(0)}, v_{\mathrm{K}}^{(0)}, \eta_{\mathrm{K}}^{(0)}\right)(x, y, t, T) \\
& +\left(u_{i_{\mathrm{IG}}}^{(0)}, v_{\mathrm{IG}}^{(0)}, \eta_{\mathrm{IG}}^{(0)}\right)(x, y, t, T) \\
& +\left(0, v_{\mathrm{M}}^{(0)}, \eta_{\mathrm{M}}^{(0)}\right)(x, T),
\end{aligned}
$$

and look for the first-order corrections - solutions of the system (20), taking care of the resonant contributions along the lines of Sect. 3.1. Note that, according to our scaling, the amplitude of the mean current is of the same order as that of the impinging wave, i.e. small. We will show later how this constraint may be relaxed.

The expressions for the resonant contributions to $R_{v}^{+}$and $R_{\eta}^{+}$in the case of wave-meanflow resonance are (cf. (21) and (22)):

$$
\begin{aligned}
R_{v}^{\mathrm{WM}^{+}} & =-c_{ \pm} \mathrm{K}_{T}^{+} e^{-x / c_{+}}-a_{+} c_{+}^{2} \mathrm{~K}^{+} \mathrm{K}_{\xi^{+}}^{+} e^{-2 x / c_{+}} \\
& -\left[\mathrm{NL}_{v}^{\mathrm{WM}^{+}} e^{i l \xi^{+}}+\text {c.c. }\right] \\
& -c_{+}\left(a_{+} v_{\mathrm{M}}^{+}+a_{-} v_{\mathrm{M}}^{-}\right) \mathrm{K}_{\xi^{+}}^{+} e^{-x / c_{+}},
\end{aligned}
$$

$$
\begin{aligned}
R_{\eta}^{\mathrm{WM}^{+}} & =\mathrm{K}_{T}^{+} e^{-x / c_{+}}+2 a_{+} c_{+} \mathrm{K}^{+} \mathrm{K}_{\xi^{+}}^{+} e^{-2 x / c_{+}} \\
& -\left[\mathrm{NL}_{\eta}^{\mathrm{WM}^{+}} e^{i l \xi^{+}}+\text {c.c. }\right] \\
& -\left(a_{+}\left(c_{+} \eta_{\mathrm{M}}^{+}-v_{\mathrm{M}}^{+}\right)+a_{-}\left(c_{+} \eta_{\mathrm{M}}^{-}-v_{\mathrm{M}}^{-}\right)\right) \mathrm{K}_{\xi^{+}}^{+} e^{-x / c_{+}},
\end{aligned}
$$

with nonlinear terms $\mathrm{NL}_{v}^{\mathrm{WM}^{+}}$and $\mathrm{NL}_{\eta}^{W M^{+}}$resulting from the wave-meanflow interaction:

$$
\begin{aligned}
\mathrm{NL}_{v}^{\mathrm{WM}^{+}} & =a_{+}\left(u_{\mathrm{IG}}^{-} \partial_{x} v_{\mathrm{M}}^{-}+v_{\mathrm{M}}^{-} \partial_{y} v_{\mathrm{IG}}^{-}\right) \\
& +a_{-}\left(u_{\mathrm{IG}}^{-} \partial_{x} v_{\mathrm{M}}^{+}+v_{\mathrm{M}}^{+} \partial_{y} v_{\mathrm{IG}}^{-}\right) \\
\mathrm{NL}_{\eta}^{\mathrm{WM}^{+}} & =a_{+}\left[\partial_{x}\left(\eta_{\mathrm{M}}^{-} u_{\mathrm{IG}}^{-}\right)+\partial_{y}\left(\eta_{\mathrm{M}}^{-} v_{\mathrm{IG}}^{-}+\eta_{\mathrm{IG}}^{-} v_{\mathrm{M}}^{+}\right)\right] \\
& +a_{-}\left[\partial_{x}\left(\eta_{\mathrm{M}}^{+} u_{\mathrm{IG}}^{-} \eta_{\mathrm{IG}}^{-} v_{\mathrm{M}}^{+}\right)+\partial_{y}\left(\eta_{\mathrm{M}}^{+} v_{\mathrm{IG}}^{-}+\eta_{\mathrm{IG}}^{-} v_{\mathrm{M}}^{+}\right)\right]
\end{aligned}
$$

Note the contributions from the Kelvin wave-meanflow interactions containing $\partial_{\xi+} \mathrm{K}^{+}$in Eqs. (40) and (41), which were absent in the case of wave-wave resonance in Sect. 3.1.

As in Sect. 3.1, in order to obtain the evolution equation for the Kelvin wave $\mathrm{K}^{+}$, we construct the combination

$$
\begin{aligned}
& \mathcal{R}=R_{\eta}^{\mathrm{WM}^{+}}-c_{+}^{-1} R_{v}^{\mathrm{WM}^{+}}=2 \mathrm{~K}_{T}^{+} e^{-x / c_{+}}+3 c_{+} \mathrm{K}^{+} \mathrm{K}_{\xi^{+}}^{+} e^{-2 x / c_{+}} \\
& +\left[2\left(a_{+} v_{\mathrm{M}}^{+}+a_{-} v_{\mathrm{M}}^{-}\right)-c_{+}\left(a_{+} \eta_{\mathrm{M}}^{+}+a_{-} \eta_{\mathrm{M}}^{-}\right)\right] \mathrm{K}_{\xi^{+}}^{+} e^{-x / c_{+}}+\mathcal{R}^{\mathrm{WM}},
\end{aligned}
$$

and require that

$$
\int_{0}^{\infty} \mathrm{d} x e^{-x / c+} \mathcal{R}=0,
$$

cf. Eq. (25). Taking into account the relations (34) between velocities and thicknesses for the mean currents, the expression for $\mathcal{R}^{\mathrm{WM}}$ is:

$$
\begin{aligned}
\mathcal{R}^{\mathrm{WM}}= & \frac{1-r}{4 c_{+}}\left(u_{\mathrm{IG}}^{-} \partial_{x x}^{2} \eta_{1_{\mathrm{M}}}+\partial_{y} v_{\mathrm{IG}}^{-} \partial_{x} \eta_{1_{\mathrm{M}}}\right) \\
& -\partial_{x}\left[u_{\mathrm{IG}}^{-}\left(\eta_{1_{\mathrm{M}}}-\eta_{2_{\mathrm{M}}}\right)\right] \\
& -\partial_{y}\left[v_{\mathrm{IG}}^{-}\left(\eta_{1_{\mathrm{IG}}}-\eta_{2_{\mathrm{IG}}}\right)+\frac{1-r}{4} \eta_{\mathrm{IG}}^{-} \partial_{x} \eta_{1_{\mathrm{M}}}\right]+c . c . .
\end{aligned}
$$

For the relevant case of an upper-layer current (35) this expression becomes:

$$
\begin{aligned}
\mathcal{R}_{\text {(upper) }}^{\mathrm{WM}} & =\frac{1-r}{4 c_{+}}\left(u_{\mathrm{IG}}^{-} \partial_{x x}^{2} \eta_{1_{\mathrm{M}}}+\partial_{y} v_{\mathrm{IG}}^{-} \partial_{x} \eta_{1_{\mathrm{M}}}\right) \\
& -(1+r) \partial_{x}\left(u_{\mathrm{IG}}^{-} \eta_{1_{\mathrm{M}}}\right) \\
& -\partial_{y}\left[(1+r) v_{\mathrm{IG}}^{-} \eta_{1_{\mathrm{IG}}}+\frac{1-r}{4} \eta_{\mathrm{IG}}^{-} \partial_{x} \eta_{1_{\mathrm{M}}}\right]+c . c . .
\end{aligned}
$$

Applying Eq. (45) we get, as in Sect. 3.1, an evolution equation for $K$ of the form:

$\mathrm{K}_{T}^{+}+C \mathrm{~K}_{\xi^{+}}^{+}+a_{+} c_{+} \mathrm{K}^{+} \mathrm{K}_{\xi^{+}}^{+}=\mathcal{S}$,

where $C$ is the non-dimensional phase velocity induced by the mean current, and dependent on the profile of the mean flow, see Eq. (44), while $\mathcal{S}$ is the source term proportional to the amplitude of the IG wave and harmonic in $\xi^{+}$. Equation (48) is of the same form as Eq. (26), apart from the linear term $\mathrm{CK}_{\xi^{+}}^{+}$, which represents a Doppler shift in the phase velocity of the Kelvin wave due to the presence of the mean current. Note that by integrating by parts, the integral in $x$ of the second term in the r.h.s. of Eq. (44) may be transformed to an integral over the mean-flow velocity minus the value of the thickness at the coast (up to a factor). Positive coastal currents will thus have lesser $C$, and vice-versa, as it should be, because Kelvin waves propagate in a negative direction in $y$. As to the efficiency of the forcing $\mathcal{S}$, it depends both on the characteristics of the mean flow and on the parameters of the impinging IG wave. For the coastal currents of finite width 
$D$, the integration over $x$ in Eq. (45) is over the finite interval $[0, D]$. From standard steepest descent estimates, e.g. Fedoryuk (1977), applied to such integrals in $\mathcal{S}$ which contain $e^{ \pm i k x}$, one can deduce that the forcing will be optimal for IG waves with $k D \leq 1$, and negligible for $k D \gg 1$. The Doppler shift term in Eq. (48) may be removed by the change of the dependent variable $K \rightarrow K-C$, and essentially it does not change the properties of the equation (48), as compared to Eq. (26). Thus the same conclusions, as to the behaviour of its solutions, hold, cf. Reznik and Zeitlin (2009). (We should remind the reader that a harmonically forced Hopf equation (26) may be fully integrated by introducing the Lagrangian coordinate $\xi^{+} \rightarrow \Xi^{+}: \dot{\Xi}^{+} \equiv\left(\partial_{t}+K \partial_{\xi^{+}}\right) \Xi^{+}$, which transforms the equation into the equation of nonlinear pendulum $\ddot{\Xi}^{+}=S\left(\Xi^{+}\right)$). That is, the amplitude of the barotropic Kelvin wave starts growing due to the resonant forcing produced by the interaction of free baroclinic IG and the coastal current. The nonlinear evolution of the thus excited Kelvin wave results, in general, in breaking and formation of characteristic mixing zones - Kelvin fronts. Yet the presence of the Doppler shift term may lead to some modifications, e.g. in the properties of stationary solutions which exist for certain initial conditions. ${ }^{2}$

\section{Conclusions and discussion}

Thus, we have shown that new mechanisms of resonant excitation of Kelvin waves are possible in the two-layer model, as compared to the barotropic one-layer one. Perhaps the most interesting one is the excitation of a barotropic Kelvin wave by a free inertia-gravity wave impinging upon a coastal current, as such a situation is ubiquitous in the ocean. As we have shown, for efficient generation, the wavelength of the incoming inertia-gravity wave should be comparable with the width of the coastal current, i.e. the wave should be long enough.

Although the calculations above were displayed in the case where the amplitude of the wave and the magnitude of the mean current are both small, following the argument of the previous work on resonant wave excitation in the presence of the mean equatorial current Reznik and Zeitlin (2007), one can easily show that if the magnitude of the current is much larger than that of the wave, but not too large, say of the order $\epsilon^{-\frac{1}{2}}$, the same evolution Eq. (48) results, but with a "faster" slow time $T_{\frac{1}{2}}=\epsilon^{\frac{1}{2}} t$. In this case, at the first two orders of the asymptotic expansion in $\epsilon^{\frac{1}{2}}$, the equation for the mean current (order $\epsilon^{-\frac{1}{2}}$ ) and the wave equation (order $\epsilon^{0}$ ) result, respectively. The crucial fact at the second order is that the mean balanced current is an exact solution of the full system. The wave-meanflow resonance and resulting evolution Eq. (48) in $T_{\frac{1}{2}}$ for $K$ arise at the third order $\epsilon^{\frac{1}{2}}$.

\footnotetext{
${ }^{2}$ I am grateful to one of the referees for this remark.
}

In order to illustrate the main ideas, the new mechanisms of the resonant excitation were presented above in the simplest configuration of layers of equal depth and a density ratio of order one. If the two-layer model is applied to the ocean, the density ratio $r$ should be taken to be close to unity, and therefore $c_{-}$becomes very small. At the same time, the upper layer should be shallower than the lower one. Corresponding changes may be done in the above-derived expressions, yet the realistic situation of a continuously stratified ocean is much more interesting for applications. As is well-known (e.g. Gill, 1982), by separation of variables in the vertical and horizontal directions in the continuously stratified primitive equations, one arrives at a solution of the linearized equations in the form of a series of baroclinic modes. For each baroclinic mode the dispersion relation is analogous to the rotating shallow water one, with the phase velocity depending on the vertical structure. Therefore, it is possible to satisfy the resonance conditions of the above-discussed types for frequency and the horizontal wavenumber. However, compatibility with vertical structure implies additional constraints. The results of the corresponding analysis will be presented elsewhere.

Acknowledgements. This work was supported by French ANR grant "SVEMO". I am grateful to both Referees for careful reading of the manuscript and useful suggestions and corrections.

Edited by: V. Shrira

Reviewed by: E. Benilov and one anonymous referee

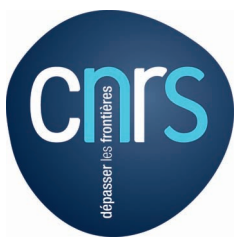

The publication of this article is financed by CNRS-INSU.

\section{References}

Fedorov, A. V. and Melville, W. K.: On the propagation and breaking of nonlinear Kelvin waves, J. Phys. Oceanogr., 25, 25192531, 1995.

Fedoryuk, M. V.: Method of steepest descent, Nauka, Moscow, 1977.

Gill, A. E.: Atmosphere-Ocean Dynamics, AP, London, 1982.

LeCunuder, A.: Excitation résonnante des ondes de Kelvin côtières dans le modèle Saint-Venant à deux couches en rotation, Master thesis, University P. and M. Curie, Paris, unpublished, 2013.

Reznik, G. M. and Zeitlin, V.: Resonant excitation and nonlinear evolution of waves in the equatorial waveguide in the presence of the mean current, Phys. Rev. Lett., 99, 064501, doi:10.1103/PhysRevLett.99.064501, 2007.

Reznik, G. M. and Zeitlin, V.: Resonant excitation of coastal Kelvin waves by inertia-gravity waves, Phys. Lett. A, 373, 1019-1021, 2009. 